Pacific Journal of Mathematics

FIXED POINT THEOREMS FOR NONEXPANSIVE MAPPINGS 


\title{
FIXED POINT THEOREMS FOR NONEXPANSIVE MAPPINGS
}

KOK-KEONG TAN

\begin{abstract}
The notions of nonexpansive, contractive, iteratively contractive and strictly contractive mappings have been generalized to a Hausdorff topological space whose topology is generated by a family of pseudometrics. A fixed point theorem for strictly contractive mappings is obtained which generalizes the Banach's contractive mapping principle. Several examples and an implicit function theorem are given as well as some applications in solving functional equations in topological vector spaces.

For iteratively contractive mappings, some results obtained by D. D. Ang and E. D. Daykin, S. C. Chu and J. B. Diaz, by M. Edelstein, by K. W. Ng and by E. Rakotch respectively are generalized.
\end{abstract}

1. Definitions and Notations. Throughout this paper $X$ is a Hausdorff topological space whose topology is generated by a family $\left\{d_{\lambda}\right\}_{\lambda \in \Gamma}$ of pseudometrics on $X$. It is well known that in order for $X$ to be such a space, it is necessary and sufficient that $X$ be a Hausdorff uniform space, or equivalently a Hausdorff completely regular space. It is clear that for any $x, y \in X$, if $x \neq y$, then there is an $\lambda \in \Gamma$ such that $d_{\lambda}(x, y)>0$. We shall denote by $\Im^{+}$the set of all nonnegative integers, $\mathfrak{R}$ the set of all natural numbers, $\Re$ the set of real numbers and $\mathfrak{S}$ the set of all complex numbers.

Notation 1.1. If $f, g: X \rightarrow X$, we shall denote by $f g$ the composition $f \circ g$ of $f$ and $g$. If $n \in \mathfrak{\Im}^{+}$, we shall denote $f^{n+1}=f^{n}(f)$, where $f^{0}=I$, the identity mapping of $X$.

Notation 1.2. If $A \subset X$ is nonempty, for each $\lambda \in \Gamma$, we denote $d_{\lambda}(A)=\sup \left\{d_{\lambda}(x, y): x, y \in A\right\}$, which is called the diameter of $A$ w.r.t. $d_{\lambda}$.

DEFINITION 1.3. If $f: X \rightarrow X$, then

(i) $f$ is nonexpansive w.r.t. $\left\{d_{\lambda}\right\}_{\lambda \in \Gamma}$ if and only if for each $\lambda \in \Gamma$, $d_{\lambda}(f(x), f(y)) \leqq d_{\lambda}(x, y)$, for all $x, y \in X$.

(ii) $f$ is contractive w.r.t. $\left\{d_{\lambda}\right\}_{\lambda \in \Gamma}$ if and only if $f$ is nonexpansive w.r.t. $\left\{d_{\lambda}\right\}_{\lambda \in \Gamma}$ and for any $x, y \in X$, if $x \neq y$, then there is a $\lambda \in \Gamma$ such that $d_{\lambda}(f(x), f(y))<d_{\lambda}(x, y)$. 
(iii) $f$ is iteratively contractive w.r.t. $\left\{d_{\lambda}\right\}_{\lambda \in \Gamma}$ if and only if $f$ is nonexpansive w.r.t. $\left\{d_{\lambda}\right\}_{\lambda \in \Gamma}$ and for any $x, y \in X$, if $x \neq y$, there is a $\lambda \in \Gamma$ and there is an $n \in \mathfrak{N}$ such that $d_{\lambda}\left(f^{n}(x), f^{n}(y)\right)<d_{\lambda}(x, y)$.

(iv) $f$ is strictly contractive w.r.t. $\left\{d_{\lambda}\right\}_{\lambda \in \Gamma}$ if and only if for each $\lambda \in \Gamma$, there is a $C_{\lambda} \in \Re$ with $0 \leqq C_{\lambda}<1$ such that $\left.d_{\lambda}(f(x)), f(y)\right) \leqq$ $C_{\lambda} d_{\lambda}(x, y)$, for all $x, y \in X$.

(v) $f$ is an isometry w.r.t. $\left\{d_{\lambda}\right\}_{\lambda \in \Gamma}$, if and only if for each $\lambda \in \Gamma$, $d_{\lambda}(f(x), f(y))=d_{\lambda}(x, y)$, for all $x, y \in X$.

By choosing an appropriate basis for the uniformity generated by the family $\left\{d_{\lambda}\right\}_{\lambda \in \Gamma}$ of pseudometrics, Definition 1.3 (i) of nonexpansiveness reduces to the notion of contraction defined by T. A. Brown and W. W. Comfort in [3], while Definition 1.3 (ii) of contractiveness reduces to the notion of $\beta$-contractiveness defined by W. J. Kammerer and R.H. Kasriel in [9]. Also Definition 1.3 (ii) is a condition used by D. D. Ang and D. E. Daykin in Theorem 1 of [1].

It is clear that if $f: X \rightarrow X$ is nonexpansive (respectively contractive, iteratively contractive or strictly contractive) w.r.t. $\left\{d_{\lambda}\right\}_{\lambda \in \Gamma}$, then for each $n \in \mathfrak{N}, f^{n}$ is nonexpansive (respectively contractive, iteratively contractive, or strictly contractive) w.r.t. $\left\{d_{\lambda}\right\}_{\lambda \in \Gamma}$. It is also clear that every strictly contractive mapping w.r.t. $\left\{d_{\lambda}\right\}_{\lambda \in \Gamma}$ is contractive w.r.t. $\left\{d_{\lambda}\right\}_{\lambda \in \Gamma}$, every contractive mapping w.r.t. $\left\{d_{\lambda}\right\}_{\lambda \in \Gamma}$ is iteratively contractive w.r.t. $\left\{d_{\lambda}\right\}_{\lambda \in \Gamma}$ and every nonexpansive mapping w.r.t. $\left\{d_{\lambda}\right\}_{\lambda \in \Gamma}$ is continuous.

If $f: X \rightarrow X$ is nonexpansive (respectively contractive, iteratively contractive or strictly contractive) w.r.t. $\left\{d_{\lambda}\right\}_{\lambda \in \Gamma}$ and if $X$ is metrizable, it is not known whether there exists a metric $d$ on $X$ inducing the same topology on $X$ such that $f$ is nonexpansive (respectively contractive, iteratively contractive or strictly contractive) w.r.t. $\{d\}$.

Notation 1.4. If $f: X \rightarrow X, X^{f}=\left\{x \in X:\right.$ there is an $x_{0} \in X$ such that $x$ is a cluster point of $\left.\left(f^{n}\left(x_{0}\right)\right)_{n=0}^{\infty}\right\}$.

In case $\left\{d_{\lambda}\right\}_{\lambda \in \Gamma}$ contains a single metric, the above notation $X^{f}$ was first introduced by M. Edelstein in [7].

Definition 1.5. Let $\left(x_{n}\right)_{n=0}^{\infty}$ be a sequence in $X$. Then $\left(x_{n}\right)_{n=0}^{\infty}$ is Cauchy if and only if for each $\lambda \in \Gamma, d_{\lambda}\left(x_{n}, x_{m}\right) \rightarrow 0$ as $n, m \rightarrow \infty$.

DEFINITION 1.6. $X$ is sequentially complete if and only if every Cauchy sequence in $X$ converges to some element in $X$.

It is known that $X$ is sequentially compact implies $X$ is countably compact and $X$ is countably compact implies $X$ is sequentially complete.

2. Strictly contractive mappings. In this section the well known 
Banach's contraction mapping principle is generalized.

Proposition 2.1. Let $f: X \rightarrow X$ be iteratively contractive w.r.t. $\left\{d_{\lambda}\right\}_{\lambda \in \Gamma}$. Then a fixed point of $f$, whenever it exists, is unique. Moreover, $x \in X$ is a fixed point of $f$ if and only if $x$ is a periodic point of $f$.

Proof. Suppose there were $\zeta, \eta \in X$ such that $f(\zeta)=\zeta \neq \eta=f(\eta)$. Since $f$ is iteratively contractive w.r.t. $\left\{d_{\lambda}\right\}_{\lambda \in \Gamma}$ and $\zeta \neq \eta$, there is an $\lambda \in \Gamma$ and there is an $n \in \mathfrak{N}$ such that $d_{\lambda}\left(f^{n}(\zeta), f^{n}(\eta)\right)<d_{\lambda}(\zeta, \eta)$, which contradicts the fact that $f^{n}(\zeta)=\zeta$ and $f^{n}(\eta)=\eta$.

Next suppose $x \in X$ is a periodic point of $f$, then $x=f^{N}(x)$, for some $N \in \mathfrak{N}$, then $x$ is a fixed point of $f^{N}$. Since $f^{N}$ is also iteratively

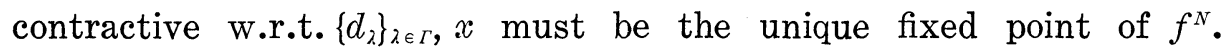
Since $f\left(f^{N}(x)\right)=f^{N}(f(x))=f(x)$, we must have $x=f(x)$. Hence $x$ is a fixed point of $f$.

If card $(\Gamma)=1$, Theorem 1 of $\mathrm{K} . \mathrm{W} . \mathrm{Ng}$ in [11] shows that the above proposition still holds even if the nonexpansiveness is dropped in defining an iteratively contractive mapping. However in a Hausdorff locally convex space, we have the following generalization:

Proposition 2.2. Let $E$ be a Hausdroff locally convex space $\left(T_{2}-\right.$ 1.c.s.), $K \subset E$, and $\mathscr{U}$ be a base for closed absolutely convex neighborhoods of 0. For each $U \in \mathscr{Q}$ let $P_{U}$ be the gauge of $U$. Suppose $f: K \rightarrow K$ is such that for any $x, y \in K$ and $U \in \mathscr{C}$, if $P_{U}(x-y)>0$, then there is an $n \in \mathfrak{N}$ such that $P_{U}\left(f^{n}(x)-f^{n}(y)\right)<P_{U}(x-y)$. Then for any $x \in K, x$ is a fixed point of $f$ if and only if $x$ is a periodic point of $f$.

Proof. Suppose $x \in K$ is a periodic point of $f$ and $f(x) \neq x$. Let $N=\inf \left\{n \in \mathfrak{N}: f^{n}(x)=x\right\}$, then $N>1$. If $f^{n}(x)=f^{n+1}(x)$ for some $n \in\{0,1, \cdots, N-1\}$, then $x=f^{N}(x)=f^{N-n}\left(f^{n}(x)\right)=f^{N-n}\left(f^{n+1}(x)\right)=$ $f^{N+1}(x)=f(x)$, which is a contradiction. Hence $f^{n}(x) \neq f^{n+1}(x)$ for any $n \in\{0,1,2, \cdots, N-1\}$. Thus $0 \notin\left\{f^{n}(x)-f^{n+1}(x): n=0,1, \cdots, N-1\right\}$. Since $\left\{f^{n}(x)-f^{n+1}(x): n=0,1, \cdots, N-1\right\}$ is closed, there exists a $U \in \mathscr{Q}$ such that $U \cap\left\{f^{n}(x)-f^{n+1}(x): n=0,1 \cdots, N-1\right\}=\varnothing$. It follows that $P_{U}\left(f^{n}(x)-f^{n+1}(x)\right)>1>0$ for each $n \in\{0,1, \cdots, N-1\}$. Since $P_{U}(x-f(x))>0$, there is an $m \in \mathfrak{N}$ such that $P_{U}\left(f^{m}(x)-f^{m+1}(x)\right)<$ $P_{U}(x-f(x))$. Let $N_{1}=\inf \left\{n \in \Re: P_{U}(x-f(x))>P_{U}\left(f^{n}(x)-f^{n+1}(x)\right)\right\}$. If $N_{1} \geqq N$, say $N_{1}=p N+q$, where $0 \leqq q<N \leqq N_{1}$ so that $P_{U}(x-f(x))>P_{U}\left(f^{N_{1}}(x)-f^{N_{1}+1}(x)\right)=P_{U}\left(f^{q}(x)-f^{q+1}(x)\right)$, which contradicts the minimality of $N_{1}$. Hence we must have $N_{1}<N$. Suppose $N_{1}, N_{2}, \cdots, N_{i} \in \mathfrak{N}$ have been defined such that for each $j=1, \cdots, i$, $N_{j}<N$ and $\left.P_{U}\left(f^{N_{j+1}}(x)-f^{N_{j+1}+1}(x)\right)<P_{U} f^{N_{j}}(x)-f^{N_{j}+1}(x)\right)$ for each 
$j=1, \cdots, i-1$. Then since $P_{U}\left(f^{N_{i}}(x)-f^{N_{i}+1}(x)\right)>0$, there is an $n \in \mathfrak{N}$ such that $P_{U}\left(f^{N_{i}}(x)-f^{N_{i}+1}(x)\right)>P_{U}\left(f^{n}\left(f^{N_{i}}(x)\right)-f^{n}\left(f^{N_{i}+1}(x)\right)\right)$. Let $N_{i+1}=\inf \left\{n \in \mathfrak{R}: P_{U}\left(f^{N i}(x)-f^{N_{i}+1}(x)\right)>P_{U}\left(f^{n}(x)-f^{n+1}(x)\right)\right\}$. If $N_{i+1} \geqq N$, say $N_{i+1}=p N+q$, where $0 \leqq q<N, p \geqq 1$, then $P_{U}\left(f^{N_{1}}(x)-\right.$ $\left.f^{N_{1}+1}(x)\right)>\cdots>P_{U}\left(f^{N_{i}}(x)-f^{N_{i}+1}(x)\right)>P_{U}\left(f^{N_{i+1}}(x)-f^{N_{i+1}+1}(x)\right)=$ $P_{U}\left(f^{q}(x)-f^{q+1}(x)\right)$, which conradicts the minimality of $N_{1}$. Hence we must have $N_{i+1}<N$. Therefore by induction there is an infinite sequence $\left(N_{i}\right)_{i=1}^{\infty}$ of positive integers such that (i) $N_{i}<N$, for all $i=1,2, \cdots$, and (ii) $P_{U}\left(f^{N_{i}}(x)-f^{N_{i+1}}(x)\right)>P_{U}\left(f^{N_{i+1}}(x)-f^{N_{i+1}+1}(x)\right)$ for all $i=1,2, \cdots$. By (i), there exist $i, j \in \mathfrak{N}$ such that $i \neq j$ while $N_{i}=N_{j}$, which contradicts (ii). Thus we must have $f(x)=x$.

The proof of the following theorem is the same as the classical Banach fixed point theorem, and is therefore omitted.

Theorem 2.3. Let $X$ be sequentially complete. If $f: X \rightarrow X$ is strictly contractive w.r.t. $\left\{d_{\lambda}\right\}_{\lambda \in \Gamma}$, then $f$ has a unique fixed point $\zeta \in X$ such that $\zeta=\lim _{n \rightarrow \infty} f^{n}(x)$, for all $x \in X$.

Proposition 2.4. Let $Y$ be any topological space and $f: Y \rightarrow Y$ (not necessarily continuous). If there exists an $N \in \mathfrak{N}$ and there is a $\zeta \in Y$ such that for each $y \in Y, \zeta=\lim _{n \rightarrow \infty}\left(f^{N}\right)^{n}(y)$, then $\zeta=\lim _{n \rightarrow \infty} f^{n}(\mathrm{y})$, for each $y \in Y$.

Proof. Let $V$ be any neighborhood of $\zeta$. If $y \in Y$, then for each $k \in\{1, \cdots, N-1\}, \zeta=\lim _{n \rightarrow \infty}\left(f^{N}\right)^{n}\left(f^{k}(y)\right)$, so that for each $k \in\{1, \cdots$, $N-1\}$, there is an $n_{k} \in \mathfrak{R}$ such that for all $n \geqq n_{k},\left(f^{N}\right)^{n}\left(f^{k}(y)\right) \in V$. Take $n_{0}=\max \left\{n_{1}, \cdots, n_{N-1}\right\}$. Then for all $n \geqq n_{0}, n \geqq n_{k}$ for all $k=1, \cdots, N-1$, so that $\left(f^{N}\right)^{n}\left(f^{k}(y)\right) \in V$ for all $n \geqq n_{0}$ and for all $k=1, \cdots, N-1$. Hence $f^{n}(y) \in V$ for all $n \geqq n_{0} N$. Thus $\zeta=\lim _{n \rightarrow \infty} f^{n}(y)$ for each $y \in Y$.

Theorem 1.3 on pp. 8 of Bonsall in [2] is a special case of the following.

CoRollary 2.5. Let $X$ be sequentially complete, $f: X \rightarrow X$ (not necessarily continuous). If there is an $N \in \mathfrak{N}$ such that $f^{N}$ is strictly contractive w.r.t. $\left\{d_{\lambda}\right\}_{\lambda \in \Gamma}$, then $f$ has a unique fixed point $\zeta \in X$ and $\zeta=\lim _{n \rightarrow \infty} f^{n}(x)$, for all $x \in X$.

Proof. By Theorem 2.3, $f^{N}$ has a unique fixed point $\zeta \in X$ such that $\zeta=\lim _{n \rightarrow \infty}\left(f^{N}\right)^{n}(x)$, for all $x \in X$. By proposition $2.4, \zeta=\lim _{n \rightarrow \infty} f^{n}(x)$ for all $x \in X$. Since $f(\zeta)=f\left(f^{N}(\zeta)\right)=f^{N}(f(\zeta))$, we must have $f(\zeta)=\zeta$.

Corollary 2.6. Let $X$ be sequentially complete, $f: X \rightarrow X$ (not necessarily continuous). If there are $R, S: X \rightarrow X$ such that $R S=1$, 
identity mapping on $X$ and if there exists an $N \in \mathfrak{R}$ such that $S f^{N} R$ is strictly contractive w.r.t. $\left\{d_{\lambda}\right\}_{\lambda \in \Gamma}$, then $f$ has a unique fixed point $\zeta \in X$ and $\zeta=R\left(\lim _{n \rightarrow \infty}\left(S f^{n} R\right)(x)\right)$, for all $x \in X$.

Proof. Since $(S f R)^{N}=S f^{N} R$ is strictly contractive w.r.t. $\left\{d_{\lambda}\right\}_{\lambda \in \Gamma}$, Sf $R$ has a unique fixed point $\eta \in X$ and $\eta=\lim _{n \rightarrow \infty}(S f R)^{n}(x)=$ $\lim _{n \rightarrow \infty}\left(S f^{n} R\right)(x)$, for all $x \in X$, by Corollary 2.5. But then it is easy to show that $\zeta=R \eta$ is a unique fixed point of $f$, and $\zeta=$ $R\left(\lim _{n \rightarrow \infty}\left(S f^{n} R\right)(x)\right)$, for all $x \in X$.

The above corollary generalizes a result of S. C. Chu and J.B. Diaz in [4].

CoRollary 2.7. Let $X$ be sequentially complete and $F$ be a family of commuting mappings on $X$. Suppose there exists an $f \in F$ and there are $R, S: X \rightarrow X$ such that (i) $R S=I$ and (ii) for some $N \in \mathfrak{N}$, $S f^{N} R$ is strictly contractive w.r.t. $\left\{d_{\lambda}\right\}_{\lambda \in \Gamma}$. Then $F$ has a unique common fixed point.

Proof. By Corrollary 2.6, $f$ has a unique fixed point, say $\zeta \in X$. If $g \in F$, then $f(g(\zeta))=g(f(\zeta))=g(\zeta)$, so that $g(\zeta)$ is also a fixed point of $f$ implies $g(\zeta)=\zeta$. Thus $\zeta$ is the unique common fixed point of $F$.

3. Some examples and applications. First we shall give an example of a mapping which is contractive but not strictly contractive while some iterates of it is strictly contractive.

ExAmple 3.1. Let $S$ be a nonempty topological space and $C(S)$ be the set of all complex-(or real)-valued continuous functions on $S$. Let $\mathscr{C}=\{C: C$ is a nonempty compact subset of $S\}$. For each $C \in \mathscr{C}$, we define $q_{C}(f)=\sup _{x \in C}|f(x)|$, for all $f \in C(S)$. Then $q_{C}$ is a seminorm on $C(S)$ for each $C \in \mathscr{C}$. Let $F=\left\{q_{C}: C \in \mathscr{C}\right\}$. If $f \in C(S)$ is non zero then $f(x) \neq 0$ for some $x \in X$, so that $q_{\{x\}}(f)>0$. By a theorem of Robertson in [13], $C(S)$ is a Hausdorff locally convex space under the topology generated by $F$. For each $C \in \mathscr{C}$, if we define $d_{C}(f, g)=$ $q_{C}(f-g)$, for all $f, g \in C(S)$, then $d_{C}$ is a pseudometric on $C(S)$ and $\left\{d_{C}\right\}_{C \in}$ generates the same topology as $F$. First we note that $C(S)$ is complete. Define $K=\left\{f \in C(S):\|f\|_{\infty}=\sup _{x \in S}|f(x)| \leqq \frac{1}{2}\right\}$, then it is clear that $K$ is nonempty closed and convex, so that $K$ is also complete and hence sequentially complete.

(i) For each $\lambda \in \mathbb{E}$ such that $|\lambda|=1$ and each $g \in C(S)$ with $\|g\|_{\infty} \leqq \frac{1}{4}$, we define $T_{\lambda, g}: K \rightarrow K$ by $T_{\lambda, g}(f)=\lambda f^{2}+g$, for all $f \in K$. Since for each $f \in K,\left\|T_{\lambda, g}(f)\right\|_{\infty}=\left\|\lambda f^{2}+g\right\|_{\infty} \leqq|\lambda|\left\|f^{2}\right\|_{\infty}+\|g\|_{\infty}=$ $\|f\|_{\infty}^{2}+\|g\|_{\infty} \leqq \frac{1}{4}+\frac{1}{4}=\frac{1}{2}, T_{\lambda, g}$ indeed maps $K$ into $K$. If $f_{1}, f_{2} \in K$, $C \in \mathscr{C}$, then $d_{C}\left(T_{\lambda, g}\left(f_{1}\right), T_{\lambda, g}\left(f_{2}\right)\right)=q_{C}\left(T_{\lambda, g}\left(f_{1}\right)-T_{\lambda, g}\left(f_{2}\right)\right)=\sup _{x \in C} \mid f_{1}^{2}(x)-$ 
$f_{2}^{2}(x)\left|=\sup _{x \in C}\right| f_{1}(x)-f_{2}(x)|| f_{1}(x)+f_{2}(x)\left|\leqq \sup _{x \in C}\right| f_{1}(x)-f_{2}(x) \mid=d_{C}\left(f_{1}, f_{2}\right)$, so that $T_{\lambda, g}$ is nonexpansive w.r.t. $\left\{d_{C}\right\}_{C \in \mathscr{C}}$. If $f_{1}, f_{2} \in K$ and $f_{1} \neq f_{2}$, then there is an $x \in S$ such that $f_{1}(x) \neq f_{2}(x)$. Since $\left|f_{1}(x)\right| \leqq \frac{1}{2}$ and $\left|f_{2}(x)\right| \leqq \frac{1}{2}$, we must have $\left|f_{1}(x)+f_{2}(x)\right|<1$. Thus for $C=\{x\}, C \in \mathscr{C}$ and $d_{C}\left(T_{\lambda, g}\left(f_{1}\right), T_{\lambda, g}\left(f_{2}\right)\right)=\left|f_{1}^{2}(x)-f_{2}^{2}(x)\right|=\left|f_{1}(x)-f_{2}(x)\right|\left|f_{1}(x)+f_{2}(x)\right|<$ $\left|f_{1}(x)-f_{2}(x)\right|=d_{C}\left(f_{1}, f_{2}\right)$. Hence $T_{2, g}$ is contractive w.r.t. $\left\{d_{C}\right\}_{C \in \varepsilon} \cdot$ However for any $\mu \in \Re$ with $0 \leqq \mu<1$, choose any $a \in \mathfrak{R}$ such that $\mu-\frac{1}{2}<a<\frac{1}{2}$ and define $h_{1} \equiv \frac{1}{2}$ and $h_{2} \equiv a$, then $h_{1}, h_{2} \in K$, so that for any $C \in \mathscr{C}$, we see that $\mu d_{C}\left(h_{1}, h_{2}\right)=\mu\left(\frac{1}{2}-a\right)<\left(\frac{1}{2}\right)^{2}-a^{2}=d_{C}\left(T_{\lambda, g}\left(h_{1}\right)\right.$, $\left.T_{\lambda, g}\left(h_{2}\right)\right)$. Therefore $T_{\lambda, g}$ is not strictly contractive w.r.t. $\left\{d_{C}\right\}_{C \in \varepsilon}$. However if $\|g\|_{\infty}<\frac{1}{4}$ it can be easily shown that for each $C \in \mathscr{C}$, $d_{C}\left(T_{\lambda, g}^{2}\left(f_{1}\right), T_{\lambda, g}^{2}\left(f_{2}\right)\right) \leqq \mu d_{C}\left(f_{1}, f_{2}\right)$ for all $f_{1}, f_{2} \in K$, where $\mu=\frac{1}{2}+2\|g\|_{\infty}<1$, and so $T_{\lambda, g}^{2}$ is strictly contractive w.r.t. $\left\{d_{C}\right\}_{C \in \mathscr{r}}$. By Corollary 2.5, $T_{\lambda, g}$ has a unique fixed point $\zeta \in K$ and $\zeta=\lim _{n \rightarrow \infty} T_{\lambda, g}^{n}(f)$, for all $f \in K$.

(ii) Suppose $T: K \rightarrow K$ is nonexpansive w.r.t. $\left\{d_{C}\right\}_{C \in \varepsilon}$. For each $\lambda \in \mathfrak{C}$ with $|\lambda| \leqq 1$ and each $g \in C(S)$ with $\|g\|_{\infty} \leqq \frac{1}{4}$ and each $n \in \mathfrak{R}$ with $n \geqq 3$, we define $T_{\lambda, n, g}: K \rightarrow K$ by $T_{\lambda, n, g}(f)=\lambda(T f)^{n}+g$, for all $f \in K$. Then for any $C \in \mathscr{C}, d_{C}\left(T_{\lambda, n, g}\left(f_{1}\right), T_{\lambda, n, g}\left(f_{2}\right)\right) \leqq n / 2^{n-1} d_{C}\left(f_{1}, f_{2}\right)$, for all $f_{1}, f_{2} \in K$. Since $0<n / 2^{n-1}<1, T_{\lambda, n, g}$ is strictly contractive w.r.t. $\left\{d_{C}\right\}_{C \in}$, . Hence by Theorem 2.3., $T_{\lambda, n, g}$ has a unique fixed point $\zeta \in K$ such that $\zeta=\lim _{m \rightarrow \infty} T_{\lambda, n, a}^{m}(f)$, for all $f \in K$.

(iii) Suppose $T: K \rightarrow K$ is nonexpansive w.r.t. $\left\{d_{C}\right\}_{C \in \mathscr{C}}$. For each $\lambda \in \Re$ with $0<\lambda<1$, each $g \in C(S)$ with $\|g\|_{\infty} \leqq \frac{1}{4}$ and each $n \in \Re$ with $n \geqq 2$, we define $V_{\lambda, n, g}(f)=\lambda(T f)^{n}+(1-\lambda) g$, for all $f \in K$. Then for each $C \in \mathscr{C}, d_{C}\left(V_{\lambda, n, g}\left(f_{1}\right), V_{\lambda, n}\left(f_{2}\right)\right) \leqq n \lambda / 2^{n-1} d_{C}\left(f_{1}, f_{2}\right)$, for all $f_{1}, f_{2} \in k$. Since $0<n \lambda / 2^{n-1}<1, V_{\lambda, n . g}$ is strictly contractive w.r.t. $\left\{d_{C}\right\}_{C \in \varepsilon}$ and so by Theorem 2.3, $V_{\lambda, n, g}$ has a unique fixed point $\zeta \in K$ and $\zeta=$ $\lim _{m \rightarrow \infty} V_{\lambda, n, g}^{m}(f)$, for all $f \in K$.

The following result is obtained by Kirk in [10] in Banach spaces. The similar proof is omitted.

THEOREM 3.2. Let $E$ be a $T_{2}$-1.c.s. whose topology is generated by a family $\mathscr{P}$ of semi-norms on $E$, and $K \subset E$ be nonempty convex. For each $p \in \mathscr{P}$, define $d_{p}(x, y)=p(x-y)$ for all $x, y \in E$. Suppose $T: K \rightarrow K$ is nonexpansive w.r.t. $\left\{d_{p}\right\}_{p \in \mathscr{G}}$. For $a_{0}, a_{1}, \cdots, a_{n} \geqq 0, n \geqq 1$, $a_{1}>0$ and $\sum_{i=0}^{n} a_{i}=1$, define $S: K \rightarrow K$ by $S(x)=\sum_{i=0}^{n} a_{i} T^{i}(x)$, for all $x \in K$. Then for any $x \in K, S(x)=x$ if and only if $T(x)=x$.

Corresponding to Theorem 2.3, we have the following implicit function theorem which is analogous to a result of E. Dubinsky in [5].

Theorem 3.3. Suppose $X$ is bounded, i.e. $d_{\lambda}(X)<\infty$ for each 
$\lambda \in \Gamma$ and $X$ is sequentially complete. Let $S$ be any topological space and $f: X \times S \rightarrow X$ be continuous. Suppose for each $\lambda \in \Gamma$, there is a constant $C_{\lambda}$ with $0 \leqq C_{\lambda}<1$ such that $d_{\lambda}(f(x, s), f(y, s)) \leqq C_{\lambda} d_{\lambda}(x, y)$, for all $x, y \in X$ and all $s \in S$. Then there is a unique continuous mapping $T: S \rightarrow X$ such that $f(T(s), s)=T(s)$, for all $s \in S$.

Proof. For each $s \in S$, define $g_{s}: X \rightarrow X$ by $g_{s}(x)=f(x, s)$, for all $x \in X$. Then $g_{s}$ is a strictly contractive mapping w.r.t. $\left\{d_{\lambda}\right\}_{\lambda \in \Gamma}$. By Theorem 2.3., there is a unique $T(s) \in X$ such that $g_{s}(T(s))=T(s)$. Hence there is a unique mapping $T: S \rightarrow X$ such that $f(T(s), s)=T(s)$, for all $s \in S$. It remains to show that $T$ is continuous.

Fix any $x_{0} \in X$. For each $n \in \mathfrak{R}$, we define $T_{n}: S \rightarrow X$ as follows: $T_{1}(s)=f\left(x_{0}, s\right)$ and $T_{n+1}(s)=f\left(T_{n}(s), s\right)$ for all $s \in S$ and all $n \in \Re$. It is clear that $T_{1}$ is continuous and it can be shown by induction that each $T_{n}$ is continuous for $n=2,3, \cdots$.

Next we want to show that $T_{n}$ converges uniformly to $T$, i.e. for any $\varepsilon>0$ and $\lambda \in \Gamma$, there exists an $N(\lambda, \varepsilon) \in \mathfrak{R}$ such that $d_{\lambda}\left(T_{n}(s), T(s)\right)<\varepsilon / 3$, for all $n>N$ and all $s \in S$. Indeed, since $X$ is bounded $d_{\lambda}(X)<\infty$, we may choose $N(\lambda, \varepsilon) \in \mathfrak{N}$ such that $C_{\lambda}^{N} d_{\lambda}(X)<\varepsilon / 3$. Thus for $n>N$, and all $s \in S$, we see that

$$
\begin{aligned}
d_{\lambda}\left(T_{n}(s), T(s)\right) & =d_{\lambda}\left(f\left(T_{n-1}(s), s\right), f(T(s), s)\right) \\
& \leqq \\
\leqq & C_{\lambda} d_{\lambda}\left(T_{n-1}(s), T(s)\right) \\
\leqq & C_{\lambda}^{n-1} d_{\lambda}\left(T_{1}(s), T(s)\right) \\
= & C_{\lambda}^{n-1} d_{\lambda}\left(f\left(x_{0}, s\right), f(T(s), s)\right) \\
\leqq & C_{\lambda}^{n} d_{\lambda}\left(x_{0}, T(s)\right) \\
\leqq & C_{\lambda}^{n} d_{\lambda}(X) \\
\leqq & C_{\lambda}^{N} d_{\lambda}(X) \\
& <\frac{\varepsilon}{3} .
\end{aligned}
$$

Suppose $s_{\mu} \rightarrow s$ in $S$. For any $\varepsilon>0$ and $\lambda \in \Gamma$ there is an $N(\lambda, \varepsilon) \in \mathfrak{N}$ such that $d_{\lambda}\left(T_{n}(s), T(s)\right)<\varepsilon / 3$, for all $n>N$ and all $s \in S$. Since $T_{N+1}$ is continuous, there is a $\mu_{0}$ with $d_{\lambda}\left(T_{N+1}\left(s_{\mu}\right), T_{N+1}(s)\right)<\varepsilon / 3$, for all $\mu>\mu_{0}$. Hence for all $\mu>\mu_{0}$,

$$
\begin{aligned}
& d_{\lambda}\left(T\left(s_{\mu}\right), T(s)\right) \leqq d_{\lambda}\left(T\left(s_{\mu}\right), T_{N+1}\left(s_{\mu}\right)\right),+d_{\lambda}\left(T_{N+1}\left(s_{\mu}\right), T_{N+1}(s)\right) \\
& \quad+d_{\lambda}\left(T_{N+1}(s), T(s)\right)<\frac{\varepsilon}{3}+\frac{\varepsilon}{3}+\frac{\varepsilon}{3}=\varepsilon
\end{aligned}
$$

so that $T\left(s_{\mu}\right) \rightarrow T(s)$. Hence $T$ is continuous.

Theorem 3.4. and Corollary 3.5 below can be obtained as a corol- 
lary and considered as an application of Theorem II of D. D. Ang and D. E. Daykin in [1].

TheOREM 3.4. Let $\left(E, E^{\prime}\right)$ be a dual pair, $A$ be a linear operator on $E$, and $A^{\prime}$ be the adjoint of $A$ such that $A^{\prime}\left(E^{\prime}\right) \subset E^{\prime}$. Suppose $A^{\prime}$ has a family $G$ of eigenvectors $e$ in $E^{\prime}$ each of which belongs to an eigenvalue $\lambda_{e}$ of modulus less than 1 and $G$ is total over $E$. Suppose for every sequence $\left(x_{n}\right)_{n=0}^{\infty}$ in $E$ such that for each $e \in G,\left(x_{n}-x_{m}, e\right) \rightarrow 0$ as $n, m \rightarrow \infty$, there is an $x \in E$ such that for each $e \in G,\left(x_{n}-x, e\right) \rightarrow 0$ as $n \rightarrow \infty$. Then for an arbitrarity fixed $y_{0} \in E$, the equation $x=$ $A(x)+y_{0}$ has a unique solution $\zeta_{y_{0}}$ and moreover

$$
\left(A^{n}(x)+A^{n-1}\left(y_{0}\right)+\cdots+y_{0}-\zeta_{y_{0}}, e\right) \rightarrow 0
$$

as $n \rightarrow \infty$ for every $e \in G$ and every $x \in E$.

Proof. For each $e \in G$, define $d_{e}(x, y)=|(x-y, e)|$, for all $x, y \in E$, then $d_{e}$ is a pseudometric on $E$. Let $E$ have the topology generated by $\left\{d_{e}\right\}_{e \in G}$. Since $G$ is total over $E$, for any $x, y \in E$ with $x \neq y$, then $x-y \neq 0$, so that there is an $e \in G$ with $(x-y, e) \neq 0$, and so $d_{e}(x, y)>0$. Hence $E$ is Hausdorff. For an arbitrarily fixed $y_{0} \in E$, define $F(x)=$ $A(x)+y_{0}$, for all $x \in E$. For each $e \in G$,

$$
\begin{aligned}
d_{e}(F(x), F(y)) & =|(A(x)-A(y), e)|=\left|\left(x-y, A^{\prime} e\right)\right| \\
& =\left|\left(x-y, \lambda_{e} e\right)\right|=\left|\lambda_{e}\right| d_{e}(x, y) .
\end{aligned}
$$

Since $\left|\lambda_{e}\right|<1$ for each $e \in G, F$ is strictly contractive w.r.t. $\left\{d_{e}\right\}_{e \in G}$. Next by hypothesis, $E$ is sequentially complete. Hence by Theorem 2.3. $F$ has a unique fixed point $\zeta_{y_{0}} \in E$ and $\zeta_{y_{0}}=\lim _{n \rightarrow \infty} F^{n}(x)$, for all $x \in E$. Thus $\zeta_{y_{0}}$ is the unique solution of $x=A(x)+y_{0}$ and

$$
\left(A^{n}(x)+A^{n-1}\left(y_{0}\right)+\cdots+y_{0}-\zeta_{y_{0}}, e\right)=\left(F^{n}(x)-\zeta_{y_{0}}, e\right) \rightarrow 0 \text { as } n \rightarrow \infty,
$$

for all $e \in G$ and each $x \in E$.

Corollary 3.5. Let $\left(E, E^{\prime}\right)$ be a dual pair such that $E$ is $\sigma\left(E, E^{\prime}\right)$, the weak topology on $E$ determined by $E^{\prime}$, sequentially complete. Let $A$ be a linear operator on $E$ and $A^{\prime}$ be the adjoint of $A$ on $E^{\prime}$ with $A^{\prime}\left(E^{\prime}\right) \subset E^{\prime}$. Suppose $A^{\prime}$ has a family $G$ of eigenvectors $e$ in $E^{\prime}$ each of which belongs to an eigenvalue $\lambda_{e}$ of modulus less than 1. If $G$ spans $E^{\prime}$, the equation $x=A(x)+y_{0}$ has a unique solution $\zeta_{y_{0}}$ such that for each $x \in E, A^{n}(x)+A^{n-1}\left(y_{0}\right)+\cdots+A\left(y_{0}\right)+y_{0} \rightarrow \zeta_{y_{0}}$ in $\sigma\left(E, E^{\prime}\right)$.

Proof. First we note that $G$ spans $E^{\prime}$ implies $G$ is total over $E$. For each $e \in G$ define $d_{e}$ as in Theorem 3.4. Suppose $\left(x_{n}\right)_{n=0}^{\infty}$ is a sequence in $E$ such that for each $e \in G, d_{e}\left(x_{n}, x_{m}\right) \rightarrow 0$ as $n, m \rightarrow \infty$. 
For any $f \in E^{\prime}$, since $G$ spans $E^{\prime}$, there are $e_{1}, \cdots, e_{n} \in G$ and there are scalars $a_{1}, \cdots, a_{n}$ such that $f=\sum_{i=1}^{n} a_{i} e_{i}$. Thus

$$
\left(x_{k}-x_{m}, f\right)=\sum_{i=1}^{n} a_{i}\left(x_{k}-x_{m}, e_{i}\right) \rightarrow 0 \text { as } k, m \rightarrow \infty .
$$

Hence $\left(x_{n}\right)_{n=0}^{\infty}$ is a Cauchy sequence in $E$ and so there is an $x \in E$ with $\left(x_{n}-x, f\right) \rightarrow 0$ as $n \rightarrow \infty$ for each $f \in E^{\prime}$. Hence the hypothesis of Theorem 3.4. is satisfied and so there is a unique solution $\zeta_{y_{0}}$ of $x=A(x)+y_{0}$ and

$$
\left(A^{n}(x)+A^{n-1}\left(y_{0}\right)+\cdots+A\left(y_{0}\right)+y_{0}-\zeta_{y_{0}}, e\right) \rightarrow 0 \text { as } n \rightarrow \infty
$$

for each $e \in G$. But then $\left(A^{n}(x)+A^{n-1}\left(y_{0}\right)+\cdots+A\left(y_{0}\right)+y_{0}-\zeta_{y_{0}}, f\right) \rightarrow 0$ as $n \rightarrow \infty$, for each $f \in E^{\prime}$, so that $A^{n}(x)+A^{n-1}\left(y_{0}\right)+\cdots+A\left(y_{0}\right)+$ $y_{0} \rightarrow \zeta_{y_{0}}$ in $\sigma\left(E, E^{\prime}\right)$.

Theorem 3.6. Let $\left(E, E^{\prime}\right)$ be a dual pair, $A$ be a linear operator on $E$ and $A^{\prime}$ be the adjoint of $A^{\prime}$ on $E^{\prime}$ such that $A^{\prime}\left(E^{\prime}\right) \subset E^{\prime}$. Suppose $A^{\prime}$ has a family $G$ of eigenvectors $e$ in $E^{\prime}$ each of which belongs to an eigenvalue $\lambda_{e} \neq 1$ with $\left|\lambda_{e}\right| \leqq 1$. Suppose either (i) $E$ is $\sigma\left(E, E^{\prime}\right)$ sequentially complete and $G$ spans $E^{\prime}$ or (ii) $G$ is total over $E$, and for every sequence $\left(x_{n}\right)_{n=0}^{\infty}$ in $E$ such that for each $e \in G,\left(x_{n}-x_{m}, e\right) \rightarrow 0$ as $n, m \rightarrow \infty$, there is an $x \in E$ such that for each $e \in G,\left(x_{n}-x, e\right) \rightarrow 0$ as $n \rightarrow \infty$. Then for any positive integer $n>1$ and $a_{1}, \cdots, a_{n}>0$ such that $\sum_{i=1}^{n} a_{i} \leqq 1$, and any arbitrarily fixed $y_{0} \in E$, there is a unique solution of the equation $x=\sum_{i=1}^{n} a_{i} A^{i}(x)+y_{0}$.

Proof. From the proof of Corollary 3.5, condition (i) implies condition (ii). Thus we may assume that (ii) holds. For each $e \in G$, define $d_{e}$ as in Theorem 3.4. Let $E$ have the topology generated by $\left\{d_{e}\right\}_{e \in G}$, then $E$ is Hausdorff and sequentially complete. Define $F(x)=$ $\sum_{i=1}^{n} a_{i} A^{i}(x)+y_{0}$ for all $x \in E$. It remains to show that $F$ is strictly contractive w.r.t. $\left\{d_{e}\right\}_{e \in G}$. Indeed, for each $e \in G$,

$$
\begin{aligned}
d_{e}(F(x), F(y)) & =\left|\left(\sum_{i=1}^{n} a_{i} A^{i}(x)-\sum_{i=1}^{n} a_{i} A^{i}(y), e\right)\right| \\
& =\left|\sum_{i=1}^{n} a_{i}\left(x-y,\left(A^{\prime}\right)^{i} e\right)\right| \\
& =\left|\sum_{i=1}^{n} a_{i}\left(x-y, \lambda_{e}^{i} e\right)\right| \\
& =\left|\sum_{i=1}^{n} a_{i} \lambda_{e}^{i}\right||(x-y, e)| \\
& =\left|\sum_{i=1}^{n} a_{i} \lambda_{e}^{i}\right| d_{e}(x, y) .
\end{aligned}
$$

Since $\left|\sum_{i=1}^{n} a_{i} \lambda_{e}^{i}\right|<1$ for all $e \in G, F$ is strictly contractive w.r.t. $\left\{d_{e}\right\}_{e \in G}$ 
and hence by Theorem 2.3, the equation $x=\sum_{i=1}^{n} a_{i} A^{i}(x)+y_{0}$ has a unique solution.

With slight changes in the hypothesis in Theorem 3.6, the above proof works for the following:

Theorem 3.7. Let $\left(E, E^{\prime}\right)$ be a dual pair, $A$ be a linear operator on $E$, and $A^{\prime}$ be a adjoint of $A$ on $E^{\prime}$ such that $A^{\prime}\left(E^{\prime}\right) \subset E^{\prime}$. Suppose $A^{\prime}$ has a family $G$ of eigenvectors $e$ in $E^{\prime}$ each of which belongs to an eigenvalue $\lambda_{e}$ with $\left|\lambda_{e}\right| \leqq 1$. Suppose either (i) $E$ is $\sigma\left(E, E^{\prime}\right)$ sequentially complete and $G$ spans $E^{\prime}$, or (ii) $G$ is total over $E$ and for every sequence $\left(x_{n}\right)_{n=0}^{\infty}$ in $E$ such that for each $e \in G,\left(x_{n}-x_{m}, e\right) \rightarrow 0$ as $n, m \rightarrow \infty$, there is an $x \in E$ such that for each $e \in G,\left(x_{n}-x, e\right) \rightarrow 0$ as $n \rightarrow \infty$. Then for any positive integer $n, a_{1}, \cdots, a_{n} \geqq 0$ with $\sum_{i=1}^{n} a_{i}<1$, and arbitrarily fixed $y_{0} \in E$, there is a unique solution of the equation $x=\sum_{i=1}^{n} a_{i} A^{i}(x)+y_{0}$.

As an application of the above Theorem 3.6 and Theorem 3.7, we have the following:-

ExAmple 3.8. Let $A$ be a diagonalizable $n \times n$ matrix over $\sqrt{5}$, and $A^{\prime}$ be its adjoint. Suppose for each eigenvalue $\lambda$ of $A^{\prime},|\lambda| \leqq 1$, then for each positive integer $n, a_{1}, \cdots, a_{n} \geqq 0$ with $\sum_{i=1}^{n} a_{i}<1$ and any arbitrarily fixed vector $y_{0}$ (an $n$-triple), the equation

$$
x=\sum_{i=1}^{n} a_{i} A^{i}(x)+y_{0}
$$

has a unique solution.

EXAMPLE 3.9. Let $A$ be a diagonalizable $n \times n$ matrix over $\sqrt{ }$, and $A^{\prime}$ be its adjoint. Suppose for each eigenvalue $\lambda$ of $A^{\prime}, \lambda \neq 1$ and $|\lambda| \leqq 1$, then for each positive integer $n>1, a_{1}, \cdots, a_{n}>0$ with $\sum_{i=1}^{n} a_{i} \leqq 1$ and any arbitrarily fixed vector $y_{0}$, the equation $x=$ $\sum_{i=1}^{n} a_{i} A^{i}(x)+y_{0}$ has a unique solution.

Although the classical Banach contraction mapping principle can be used to prove the following, it is a special case of the above two examples.

ExAmple 3.10. Let $E$ be a finite dimensional complex Hilbert space, $A$ be a normal operator on $E$. (a) If $\|A\| \leqq 1$ and $1 \notin \sigma(A)$, the spectrum of $A$, then for any positive integer $n>1, a_{1}, \cdots, a_{n}>0$, with $\sum_{i=1}^{n} a_{i} \leqq 1$ and any arbitrarily fixed $y_{0} \in E$, the equation $x=$ $\sum_{i=1}^{n} a_{i} A^{i}(x)+y_{0}$ has a unique solution. (b) If $\|A\| \leqq 1$ then for any positive integer $n, a_{1}, \cdots, a_{n} \geqq 0$ with $\sum_{i=1}^{n} a_{i}<1$ and any arbitrarily fixed $y_{0} \in E$, the equation $x=\sum_{i=1}^{n} a_{i} A^{i}(x)+y_{0}$ has a unique solution. 


\section{Iteratively contractive mappings :}

Proposition 4.1. Suppose $f: X \rightarrow X$ is nonexpansive w.r.t $\left\{d_{\lambda}\right\}_{\lambda \in \Gamma}$. If there are $\zeta, x_{0} \in X$ such that $\zeta$ is a fixed point of $f$ and $\zeta$ is a cluster point of the sequence $\left(f^{n}\left(x_{0}\right)\right)_{n=0}^{\infty}$, then $\lim _{n \rightarrow \infty} f^{n}\left(x_{0}\right)$ exists and $\zeta=\lim _{n \rightarrow \infty} f^{n}\left(x_{0}\right)$.

Proof. For any $\varepsilon>0$ and $\lambda \in \Gamma$, there is an $N \in \mathfrak{R}$ with $d_{\lambda}\left(f^{N}\left(x_{0}\right)\right.$, $\zeta)<\varepsilon$; but then for all $n \geqq N, d_{\lambda}\left(f^{n}\left(x_{0}\right), \zeta\right) \leqq d_{\lambda}\left(f^{N}\left(x_{0}\right), \zeta\right)<\varepsilon$. Hence $\zeta=\lim _{n \rightarrow \infty} f^{n}\left(x_{0}\right)$.

The following proposition is a corollary of Proposition 1 of $\mathrm{M}$. Edelstein in [8]:-

Proposition 4.2. Let $f: X \rightarrow X$ be nonexpansive w.r.t. $\left\{d_{\lambda}\right\}_{\lambda \in \Gamma}$ and $x \in X$. Then $x \in X^{f}$ if and only if $x$ is a cluster point of $\left(f^{n}(x)\right)_{n=0}^{\infty}$.

The following proposition is a corollary of Theorem 1 of $\mathrm{M}$. Edelstein in [8]:-

Proposition 4.3. Let $f: X \rightarrow X$ be nonexpansive w.r.t. $\left\{d_{\lambda}\right\}_{\lambda \in \Gamma^{*}}$ If $x \in X^{f}$, then $f$ is an isometry on $\left(f^{n}(x)\right)_{n=0}^{\infty}$ w.r.t. $\left\{d_{\lambda\}_{\lambda \in \Gamma} \text {, i.e. for each }}\right.$ $\lambda \in \Gamma d_{\lambda}\left(f^{m+k}(x), f^{n+k}(x)\right)=d_{\lambda}\left(f^{m}(x), f^{n}(x)\right)$, for all $m, n, k \in \mathfrak{\Im}^{+}$.

THEOREM 4.4. Let $f: X \rightarrow X$ be iteratively contractive w.r.t. $\left\{d_{\lambda}\right\}_{\lambda \in \Gamma}$. Then Card $\left(X^{f}\right) \leqq 1$. In case Card $\left(X^{f}\right)=1, X^{f}$ contains only the unique fixed point of $f$.

Proof. Suppose $X^{f} \neq \varnothing$ and $x \in X^{j}$. If $f(x) \neq x$, then $f$ is iteratively contractive w.r.t. $\left\{d_{\lambda}\right\}_{\lambda \in \Gamma}$ implies there is a $\lambda_{0} \in \Gamma$ and there is an $n \in \mathfrak{N}$ such that $d_{\lambda_{0}}\left(f^{n}(x), f^{n+1}(x)\right)<d_{\lambda_{0}}(x, f(x))$. By Proposition 4.3, $f$ is an isometry on $(f(x))_{n=0}^{\infty}$ w.r.t. $\left\{d_{\lambda}\right\}_{\lambda \in \Gamma}$, and so in particular, $d_{\lambda_{0}}\left(f^{n+1}(x), f^{n}(x)\right)=d_{\lambda_{0}}\left(f^{n}(x), f^{n-1}(x)\right) \cdots=d_{\lambda_{0}}(f(x), x)$, which is a contradiction. Hence $f(x)=x$. Since any fixed point of $f$ is unique, $X^{f}=\{x\}$

COROLlary 4.5. Let $f: X \rightarrow X$ be iteratively contractive w.r.t. $\left\{d_{\lambda}\right\}_{\lambda \in \Gamma \cdot}$. Suppose there is an $x_{0} \in X$ such that $\left(f^{n}\left(x_{0}\right)\right)_{n=0}^{\infty}$ has a cluster point $\zeta \in X$, then $\zeta$ is the unique fixed point of $f$ and $\lim _{n \rightarrow \infty} f^{n}\left(x_{0}\right)=\zeta$.

Proof. Since $\zeta$ is a cluster point of $\left(f^{n}\left(x_{0}\right)\right)_{n=0}^{\infty}, \zeta \in X^{f}$. By Theorem $4.4 \zeta$ is the unique fixed point of $f$. By Proposition 4.1, $\zeta=\lim _{n \rightarrow \infty} f^{n}\left(x_{0}\right)$.

The above corollary generalizes Theorem 1 of D. D. Ang and D. E. Daykin [1]. 
Corollary 4.6. Let $f: X \rightarrow X$ be such that for some $N \in \mathfrak{R}$, $f^{N}$ is iteratively contractive w.r.t. $\left\{d_{\lambda}\right\}_{\lambda \in \Gamma}$. If for some $x_{0} \in X$, the sequence $\left(\left(f^{N}\right)^{n}\left(x_{0}\right)\right)_{n=0}^{\infty}$ has a cluster point $\zeta \in X$, then $\zeta$ is the unique fixed point of $f$ and $\zeta=\lim _{n \rightarrow \infty} f^{N n}\left(x_{0}\right)$.

Proof. By Corollary 4.5, $\zeta$ is the unique fixed point of $f^{N}$ and $\zeta=\lim _{n \rightarrow \infty} f^{N n}\left(x_{0}\right)$. Since $f(\zeta)=f\left(f^{N}(\zeta)\right)=f^{N}(f(\zeta))$, we must have $f(\zeta)=\zeta$.

COROLlaRY 4.7. Let $f: X \rightarrow X$ be iteratively contractive w.r.t. $\left\{d_{\lambda}\right\}_{2 \in \Gamma \cdot}$. If there are $\zeta, x_{0} \in X$ and there is a sequence $\left(n_{i}\right)_{i=0}^{\infty}$ in $\mathfrak{N}$ with $1 \leqq n_{1}<n_{2}<\cdots$ such that $\zeta=\lim _{i \rightarrow \infty} f^{n_{i}}\left(x_{0}\right)$, then $\lim _{n \rightarrow \infty} f^{n}\left(x_{0}\right)$ exists and $f(\zeta)=\zeta=\lim _{n \rightarrow \infty} f^{n}\left(x_{0}\right)$.

Theorem 1 of M. Edelstein in [6] is a special case of the above corollary.

COROLlaRY 4.8. If $X$ is sequentially compact or countably compact and $f: X \rightarrow X$ is such that for some $N \in \mathfrak{R}, f^{N}$ is iteratively contractive w.r.t. $\left\{d_{\lambda}\right\}_{\lambda \in \Gamma}$, then $f$ has a unique fixed point $\xi \in X$ such that $\xi=\lim _{n \rightarrow \infty} f^{n}(x)$, for all $x \in X$.

CoROLlaRy 4.9. Let $F$ be a family of commuting mappings on $X$. Suppose there exists $f \in F$, there are $R, S: X \rightarrow X$ with $R S=I$ and there is an $N \in \mathfrak{R}$ such that $S f^{N} R$ is iteratively contractive w.r.t. $\left\{d_{\lambda}\right\}_{, \in I}$. If there is an $x_{0} \in X$ such that the iterates of $x_{0}$ under $S f^{N} R$ has a cluster point in $X$, then $F$ has a unique common fixed point.

Proof. By Corollary 4.6, $\zeta=\lim _{n \rightarrow \infty} S f^{N n} R\left(x_{0}\right)$ is the unique fixed point of $S f R$. Hence $R \zeta$ is the unique fixed point of $f$. For any $g \in F$, $g(\zeta)=g(f(\zeta))=f(g(\zeta))$, and so $g(\zeta)=\zeta$. Hence $\zeta$ is the unique common fixed point for $F$.

THeOREM 4.10. Let $f: X \rightarrow X$ be iteratively contractive w.r.t. $\left\{d_{\lambda}\right\}_{\lambda \in \Gamma}$. Suppose there is an $x_{0} \in X$ such that if

$$
M_{x_{0}}=\bigcup_{\lambda \in \Gamma}\left\{x \in X: d_{\lambda}(x, f(x))<d_{\lambda}\left(x_{0}, f\left(x_{0}\right)\right)\right\},
$$

then every sequence in $M_{x_{0}}$ has a cluster point in $X$. Then $f$ has a unique fixed point $\zeta \in X$ and $\zeta=\lim _{n \rightarrow \infty} f^{n}\left(x_{0}\right)$.

Proof. Since $f$ is nonexpansive w.r.t. $\left\{d_{\lambda}\right\}_{\lambda \in \Gamma}$, we see that $f\left(M_{x_{0}}\right) \subset$ $M_{x_{0}}$. If $f\left(x_{0}\right)=x_{0}$, then $x_{0}$ is the unique fixed point of $f$ and $x_{0}=$ 
$\lim _{n \rightarrow \infty} f^{n}\left(x_{0}\right)$. Suppose $f\left(x_{0}\right) \neq x_{0}$, then there is a $\lambda \in \Gamma$ and there is an $n_{0} \in \mathfrak{N}$ such that $d_{\lambda}\left(f^{n_{0}}\left(x_{0}\right), f^{n_{0}+1}\left(x_{0}\right)\right)<d_{\lambda}\left(x_{0}, f\left(x_{0}\right)\right)$. Thus $f^{n_{0}}\left(x_{0}\right) \in M_{x_{0}}$ so that $f^{n}\left(x_{0}\right) \in M_{x_{0}}$, for all $n \geqq n_{0}$. By hypothesis, there is an $\zeta \in X$ such that $\zeta$ is a cluster point of $\left(f^{n}\left(x_{0}\right)\right)_{n \geq n_{0}}$. By Corollary $4.5 \zeta$ is the unique fixed point of $f$ and $\zeta=\lim _{n \rightarrow \infty} f^{n}\left(x_{0}\right)$.

Corollary 4.11. Let $f: X \rightarrow X$ be iteratively contractive w.r.t. $\left\{d_{\lambda}\right\}_{\lambda \in \Gamma}$. Suppose there exists an $x_{0} \in X$ and there is a subset $M$ of $X$ such that (i) $M$ is countably compact and (ii) for any $\lambda \in \Gamma$ and $x \in X \sim M, d_{\lambda}\left(x, x_{0}\right)-d_{\lambda}\left(f(x), f\left(x_{0}\right)\right) \geqq 2 d_{\lambda}\left(x_{0}, f\left(x_{0}\right)\right)$. Then $\lim _{n \rightarrow \infty} f^{n}\left(x_{0}\right)$ exists and is the unique fixed point of $f$.

Proof. Define $M_{x_{0}}=\mathbf{U}_{\lambda \in \Gamma}\left\{x \in X: d_{\lambda}(x, f(x))<d_{\lambda}\left(x_{0}, f\left(x_{0}\right)\right)\right\}$. If $x \in X \sim M$, then for any $\lambda \in \Gamma$,

$$
\begin{aligned}
& 2 d_{\lambda}\left(x_{0}, f\left(x_{0}\right)\right) \leqq \\
\leqq & d_{\lambda}\left(x, x_{0}\right)-d_{\lambda}\left(f(x), f\left(\left(x_{0}\right)\right)\right. \\
\equiv & d_{\lambda}(x, f(x))+d_{\lambda}\left(f(x), f\left(x_{0}\right)\right)+d_{\lambda}\left(f\left(x_{0}\right), x_{0}\right)-d_{\lambda}\left(f(x), f\left(x_{0}\right)\right) \\
= & d_{\lambda}(x, f(x))+d_{\lambda}\left(f\left(x_{0}\right), x_{0}\right)
\end{aligned}
$$

so that $d_{\lambda}\left(x_{0}, f\left(x_{0}\right)\right) \leqq d_{\lambda}(x, f(x))$ for all $\lambda \in \Gamma$, and hence $x \in X \sim M_{x_{0}}$. Thus $X \sim M \subset X \sim X_{x_{0}}$ and so $M_{x_{0}} \subset M$. Since the hypothesis of Theorem 4.10 is satisfied, $\lim _{n \rightarrow \infty} f^{n}\left(x_{0}\right)$ exists and is the unique fixed point of $f$. The above Corollary generalizes Theorem 1 of E. Rakotch in [12].

\section{REFERENCES}

1. D. D. Ang and D. E. Daykin, Some Fixed point theorems and convolution equations, Proc. Amer. Math. Soc., 19 (1968), 1187-1194.

2. F. F. Bonsall, Lectures on some fixed point theorems of functional analysis, Tata Institute of Fundamental Research, Bombay, India, (1962).

3. T. A. Brown and W. W. Comfort, New Method for expansion and contraction maps in uniform spaces, Proc. Amer. Math. Soc., 11 (1960), 483-486.

4. S. C. Chu and J. B. Diaz, A fixed point theorem for " in the large" application of the contraction principle, Atti Accad. Sci. Torinto, 99 (1965), 351-363.

5. E. Dubinsky, Fixed points in non-normed spaces, Ann. Acad. Sci. Fen. Series A, I. Math., 331 (1963).

6. M. Edelstein, On fixed and periodic points under contractive mappings, J. London Math. Soc., 37 (1962), 74-79.

7. - - On nonexpansive mappings, Proc. Amer. Math. Soc., 15 (1964), 689695.

8. - On nonexpansive mappings of uniform spaces, Akademic von Wetenschappen Amst. Proc. Series A. Math., 68 (1965), 47-51.

9. W. J. Kammerer and R. H. Kasriel, On contractive mappings in uniform spaces, Proc. Amer. Math. Soc., 15 (1964), 288-290.

10. W. A. Kirk, On successive approximations for nonexpansive mappings in Banach spaces, (to appear).

11. K. W. Ng, A remark on contractive mappings, Canadian Math. Bull., 13 (1970), 111-113. 
12. E. Rakotch, A note on contractive mappings, Proc. Amer. Math. Soc., 13 (1962), 459-465.

13. A. P. Robertson and W. Robertson, Topological vector spaces, Cambridge Univ. Press (1964).

Received December 10, 1970 and in revised form January 24, 1972. The preparation of this paper was partially supported by National Research Council of Canada under Grant A8096.

Dalhousie University, Halitax, Nova Scotia, Canada 


\title{
PACIFIC JOURNAL OF MATHEMATICS
}

\author{
EDITORS
}

H. SAMELSON

Stanford University

Stanford, California 94305

C. R. HOBBY

University of Washington

Seattle, Washington 98105

\section{J. DugundJI}

Department of Mathematics

University of Southern California

Los Angeles, California 90007

RICHARD ARENS

University of California

Los Angeles, California 90024

\section{ASSOCIATE EDITORS}
E. F. BeCKeNBACH
B. H. NeUmanN
F. WoLF
K. YoSHIDA

\section{SUPPORTING INSTITUTIONS}

\author{
UNIVERSITY OF BRITISH COLUMBIA \\ CALIFORNIA INSTITUTE OF TECHNOLOGY \\ UNIVERSITY OF CALIFORNIA \\ MONTANA STATE UNIVERSITY \\ UNIVERSITY OF NEVADA \\ NEW MEXICO STATE UNIVERSITY \\ OREGON STATE UNIVERSITY \\ UNIVERSITY OF OREGON \\ OSAKA UNIVERSITY
}

\author{
UNIVERSITY OF SOUTHERN CALIFORNIA \\ STANFORD UNIVERSITY \\ UNIVERSITY OF TOKYO \\ UNIVERSITY OF UTAH \\ WASHINGTON STATE UNIVERSITY \\ UNIVERSITY OF WASHINGTON

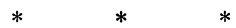 \\ AMERICAN MATHEMATICAL SOCIETY \\ NAVAL WEAPONS CENTER
}

The Supporting Institutions listed above contribute to the cost of publication of this Journal, but they are not owners or publishers and have no responsibility for its content or policies.

Mathematical papers intended for publication in the Pacific Journal of Mathematics should be in typed form or offset-reproduced, (not dittoed), double spaced with large margins. Underline Greek letters in red, German in green, and script in blue. The first paragraph or two must be capable of being used separately as a synopsis of the entire paper. The editorial "we" must not be used in the synopsis, and items of the bibliography should not be cited there unless absolutely necessary, in which case they must be identified by author and Journal, rather than by item number. Manuscripts, in duplicate if possible, may be sent to any one of the four editors. Please classify according to the scheme of Math. Rev. Index to Vol. 39. All other communications to the editors should be addressed to the managing editor, Richard Arens, University of California, Los Angeles, California, 90024.

50 reprints are provided free for each article; additional copies may be obtained at cost in multiples of 50 .

The Pacific Journal of Mathematics is published monthly. Effective with Volume 16 the price per volume (3 numbers) is $\$ 8.00$; single issues, $\$ 3.00$. Special price for current issues to individual faculty members of supporting institutions and to individual members of the American Mathematical Society: $\$ 4.00$ per volume; single issues $\$ 1.50$. Back numbers are available.

Subscriptions, orders for back numbers, and changes of address should be sent to Pacific Journal of Mathematics, 103 Highland Boulevard, Berkeley, California, 94708.

PUBLISHED BY PACIFIC JOURNAL OF MATHEMATICS, A NON-PROFIT CORPORATION

Printed at Kokusai Bunken Insatsusha (International Academic Printing Co., Ltd.), 270, 3-chome Totsuka-cho, Shinjuku-ku, Tokyo 160, Japan. 


\section{Pacific Journal of Mathematics}

\section{Vol. 41, No. $3 \quad$ BadMonth, 1972}

George E. Andrews, Two theorems of Gauss and allied identities proved arithmetically.................................. 563

Stefan Bergman, On pseudo-conformal mappings of circular domains . . . . 579

Beverly L. Brechner, On the non-monotony of dimension ............ 587

Richard Anthony Brualdi and John H. Mason, Transversal matroids and Hall's theorem .................................... 601

Philip Throop Church and James Timourian, Differentiable maps with

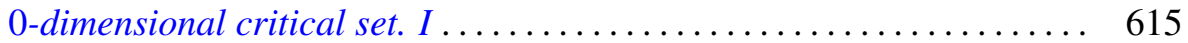

John H. E. Cohn, Squares in some recurrent sequences ............. 631

Robert S. Cunningham, Edgar Andrews Rutter and Darrell R. Turnidge, Rings of quotients of endomorphism rings of projective modules ......

Eldon Dyer and S. Eilenberg, An adjunction theorem for locally equiconnected spaces................................... 669

Michael W. Evans, On commutative P. P. rings................. 687

Ronald Lewis Graham, Hans Sylvain Witsenhausen and Hans Zassenhaus, On tightest packings in the Minkowski plane ..................

Stanley P. Gudder, Partial algebraic structures associated with

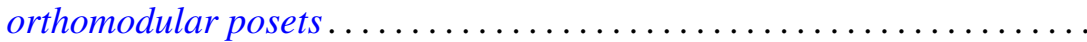

Karl Edwin Gustafson and Gunter Lumer, Multiplicative perturbation of

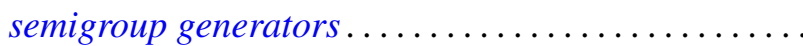

Kurt Kreith and Curtis Clyde Travis, Jr., Oscillation criteria for selfadjoint elliptic equations...

Lawrence Louis Larmore, Twisted cohomology theories and the single obstruction to lifting...........................

Jorge Martinez, Tensor products of partially ordered groups . . .

Robert Alan Morris, The inflation-restriction theorem for Amitsur cohomology ...

Leo Sario and Cecilia Wang, The class of $(p, q)$-biharmonic functions ...

Manda Butchi Suryanarayana, On multidimensional integral equations of

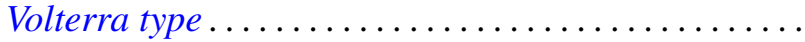

